\title{
Correction to: Hypoxic condition enhances chondrogenesis in synovium-derived mesenchymal stem cells
}

Hyun Cheol Bae, Hee Jung Park, Sun Young Wang, Ha Ru Yang, Myung Chul Lee and Hyuk-Soo Han*

\section{Correction to: Biomater Res}

https://doi.org/10.1186/s40824-018-0134-x

The original article [1] contains errors in the Acknowledgements and Funding sections of the Declarations. The two corrected sections are as follows:

\section{Acknowledgements}

Not applicable.

\section{Funding}

This study was funded by Hanmi Pharm. The funding source had no role in the study design, collection, analysis or interpretation of the data, writing of the manuscript, or in the decision to submit the manuscript for publication.

Received: 22 February 2019 Accepted: 22 February 2019

Published online: 04 March 2019

\section{Reference}

1. Bae HC, et al. Hypoxic condition enhances chondrogenesis in synovium-

derived mesenchymal stem cells. Biomater Res. 2018;22:28 https://doi.org/

10.1186/s40824-018-0134-x.

\footnotetext{
* Correspondence: oshawks7@snu.ac.kr

Department of Orthopedic Surgery, Seoul National University Hospital,

Yongondong, Chongnogu, Seoul 110-744, Republic of Korea
}

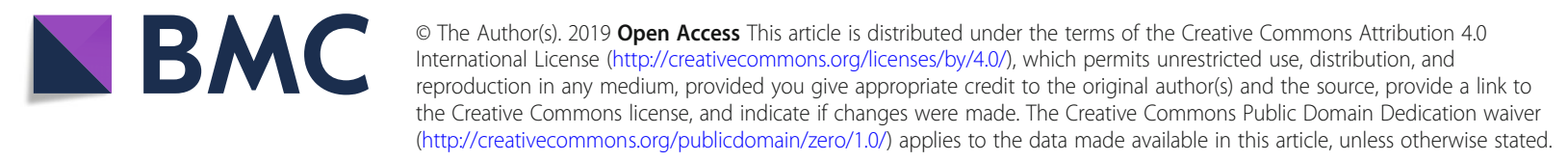

